\title{
ANALYTIC FUNCTIONS WITH SMOOTH ABSOLUTE VALUE OF BOUNDARY DATA
}

\section{F.A. SHAMOYAN}

\begin{abstract}
Let $f$ be an analytic function in the unit circle $D$ continuous up to its boundary $\Gamma, f(z) \neq 0, z \in D$. Assume that on $\Gamma$, the function $|f|$ has a modulus of continuity $\omega(|f|, \delta)$. In the paper we establish the estimate $\omega(f, \delta) \leqslant A \omega(|f|, \sqrt{\delta})$, where $A$ is a some non-negative number, and we prove that this estimate is sharp. Moreover, in the paper we establish a multi-dimensional analogue of the mentioned result. In the proof of the main theorem, an essential role is played by a theorem of Hardy-Littlewood type on Hölder classes of the functions analytic in the unit circle.
\end{abstract}

Keywords: analytic function, modulus of continuity, factorization, outer function.

Mathematics Subject Classification: primary: 30D55, 30D15; secondary: 46E22, $47 \mathrm{~A} 15$

\section{INTRODUCTION}

Let $D=\{z \in \mathbb{C}:|z|<1\}$ be a bounded unit circle in the complex plane $\mathbb{C}$ and $\Gamma$ be its boundary. By $C_{A}$ we denote the set of all functions $f$ analytic in $D$ and continuous in $D \cup \Gamma$. If $f \in C(\Gamma)$, then by the symbol $\omega(f, \delta)$ we denote the modulus of continuity of the function $f$ on $\Gamma$, that is,

$$
\omega(f, \delta)=\left\{\sup \left|f(\gamma)-f\left(\gamma e^{i t}\right)\right|, \quad \gamma \in \Gamma, \quad|t| \leqslant \delta, \quad t \in \mathbb{R}\right\} .
$$

In the paper we consider the following problem: let $f \in C_{A}$ and at the unit circle the function $\left|f\left(e^{i \theta}\right)\right|$ has the modulus of continuity $\omega(|f|, \delta)$. What is the modulus of continuity of the function $f$ on $\Gamma$ and hence, on $D \cup \Gamma$ ?

Such problem in the classes of continuous functions with the modulus of continuity satisfying Bari-Stechkin condition

$$
\int_{0}^{\delta} \frac{\omega(|f|, \delta)}{t} d t+\delta \int_{\delta}^{\pi} \frac{\omega(|f|, \delta)}{t^{2}} d t=O(\omega(|f|, \delta)), \quad \delta \rightarrow 0,
$$

was solved first in work by V.P. Khaving and the author, see [5].

It was established that if $\omega(|f|, \delta)$ satisfies Bari-Stechkin condition (1) and $f(z) \neq 0, z \in D$, then

$$
\omega(f, \delta)=O(\omega(|f|, \sqrt{\delta})), \quad \delta \rightarrow 0 .
$$

Moreover, it was shown by simple examples that the obtained estimate was sharp and the condition $f(z) \neq 0, z \in D$, is necessary in the known sense. The detailed proof of these statements was exposed in [7]. This work gave rise to rather interesting studies in this direction. First V.P. Khavin, see [6], proposed an interesting approach for obtaining such estimates; this was done by applying the methods of the theory of singular integral operators. Later N.A. Shirokov (see [8], [10]) extended the results of such type for external functions and Hölder

F.A. Shamoyan,Analytic functions with Smooth absolute value of Boundary Data.

(C) SHAMOYAN F.A. 2017.

Submitted May 10, $201 \%$. 
classes of order $\alpha, \alpha \in(0,+\infty)$ and he obtained the necessary and sufficient condition for $\left|f\left(e^{i \theta}\right)\right|$ ensuring that the function $f$ has a prescribed modulus of continuity on the set $D \cup \Gamma$. In these works there were introduced a new characteristics and in terms of this characteristics, N.A. Shirokov obtained the results of such kind also for the Besov classes of analytic functions in $D \cup \Gamma$. And finally, we mention work [2], where it was established that this phenomenon has a local character, that is, if the modulus of continuity $|f|$ on the circle satisfies the Hölder condition of order $\alpha$ just at one point, then $f$ belongs to the Hölder class of order $\frac{\alpha}{2}$ at this point.

We note that the proof by V.P. Khavni and the proof of the results in works [2], [6], [8], [10] are based on gentle and fine methods of complex and harmonic analysis. In our opinion, the approach applied in works [5] and [7] and is based on classical theorems of Hardy-Littlewood type theorems (see [3], [4]) is more simple. In this work by developing the methods of works [5], [7] we prove such results for the modulus of continuity $\omega(|f|, \delta)$ satisfying the classical Zygmund condition

$$
\int_{0}^{\delta} \frac{\omega(|f|, t)}{t} d t=O(\omega(|f|, \delta)), \quad \delta \rightarrow 0 .
$$

The following statement is true.

Theorem 1. Let $f$ be a function in the class $C_{A}$ and $f(z) \neq 0, z \in D$. If the modulus of the continuity $\omega(|f|, \delta)$ of the function $|f|$ on $\Gamma$ satisfies Zygmund condition (2), then

$$
\omega(f, \delta)=O(\omega(|f|, \sqrt{\delta})), \quad \delta \rightarrow 0,
$$

and this estimate is sharp.

Remark 1. A simple example, the function

$$
f(z)=(1-z)^{2 \alpha} \exp \left(-\frac{1+z}{1-z}\right), \quad z \in D \cup \Gamma, \quad \alpha \in(0,+\infty),
$$

where the principal branch of the power function is chosen, shows the sharpness of the statement of Theorem 1.

The analogue of Theorem 1 is true for analytic functions in the unit ball of the space $\mathbb{C}^{n}$. In order to formulate it, we introduce some notations. Let $z=\left(z_{1}, \ldots, z_{n}\right) \in \mathbb{C}^{n}$, $\|z\|=$ $\sqrt{\left|z_{1}\right|^{2}+\ldots+\left|z_{n}\right|^{2}}$. We define $B_{n}=\left\{z \in \mathbb{C}^{n}:\|z\|<1\right\}$ and $S_{n}=\left\{z \in \mathbb{C}^{n}:\|z\|=1\right\}$.

By $H\left(B_{n}\right)$ we denote the set of all analytic functions in $B_{n}$. Let $f \in H\left(B_{n}\right)$ and $f(z)=$ $\sum_{k=0}^{+\infty} f_{k}(z)$ be the expansions of the function $f$ into homogeneous polynomials; by $R(f)$ the radial derivative of the function $f[11]$, that is,

$$
R(f)(z)=\sum_{k=1}^{+\infty} k f_{k}(z), \quad z \in B_{n} .
$$

We introduce also the notation:

$$
C_{A}\left(B_{n}\right)=H\left(B_{n}\right) \cap C\left(B_{n} \cup S_{n}\right) .
$$

The following estimate of Theorem 1 is true in the classes $C_{A}\left(B_{n}\right)$.

Theorem 2. Let $f \in C_{A}\left(B_{n}\right)$ and the modulus of continuity $\omega(|f|, \delta)$ of the function $|f|$ on $S_{n}$ satisfies Zygmund condition (2). Then the modulus of continuity of the function on the set $B_{n} \cup S_{n}$ satisfies the estimate

$$
\omega(f, \delta) \leqslant A \omega(|f|, \sqrt{\delta}), \quad 0 \leqslant \delta \leqslant 2 .
$$


Remark 2. For Hölder classes, that is, as $\omega(f, t)=t^{\alpha}, 0<\alpha \leqslant 1, t \in[0,2]$, the analogue of Theorem 2 was established in work [9] by N.A. Shirokov.

\section{Proof of AUXiliary STATEMEnts}

Let $f$ and $g$ be real-valued functions with a common domain $E \subset \mathbb{C}$. Then the relation $f \lesssim g$ on $E$ is equivalent to the following: there exists a positive number $A$ such that $f(z) \leqslant A g(z)$ for each $z \in E$. If $f \lesssim g$ and simultaneously $g \lesssim f$, then $f(z) \approx g(z)$.

In what follows, as a function of modulus of continuity type we call a non-negative nondecreasing function $\omega$ on $[0,+\infty)$ such that

$$
\omega(0)=0, \quad \omega\left(\delta_{1}+\delta_{2}\right) \leqslant \omega\left(\delta_{1}\right)+\omega\left(\delta_{2}\right), \quad \omega(\lambda \delta) \leqslant 2 \lambda \omega(\delta), \quad \lambda, \delta \in[0,+\infty) .
$$

Lemma 1. Let $\omega$ be a function of modulus of continuity type satisfying Zygmund condition (2), then

$$
\omega(\delta) \ln \frac{1}{\delta} \lesssim \omega(\sqrt{\delta}), \quad \delta>0 .
$$

Proof. By the definition we have

$$
\int_{0}^{\sqrt{\delta}} \frac{\omega(u)}{u} d u \leqslant A \omega(\sqrt{\delta}) .
$$

It is clear that if $1 \leqslant \delta$, then estimate (4) is obvious and this is why we assume that $0<\delta<1$. Then

$$
\int_{0}^{\sqrt{\delta}} \frac{\omega(u)}{u} d u=\int_{0}^{\delta} \frac{\omega(u)}{u} d u+\int_{\delta}^{\sqrt{\delta}} \frac{\omega(u)}{u} d u .
$$

Hence,

$$
\int_{0}^{\sqrt{\delta}} \frac{\omega(u)}{u} d u \geqslant \omega(\delta) \int_{\delta}^{\sqrt{\delta}} \frac{d u}{u}=\frac{\omega(\delta)}{2} \ln \frac{1}{\delta} .
$$

It remains to employ Zygmund condition. The proof is complete.

Lemma 2. Let $f \in C_{A}, t=|t| \tau, t \in D, \tau \in \Gamma$. Then the estimate

$$
|f(t)| \lesssim\left(|f(\tau)|+\omega(f,(1-|t|)) \ln \frac{1}{1-|t|}\right)
$$

is true.

Proof. We have

$$
f(t)=\frac{1}{2 \pi} \int_{\Gamma} P_{t}(\xi) f(\xi)|d \xi| .
$$

where $P_{t}(\xi)$ is the Poisson kernel. This is why

$$
|f(t)| \leqslant \frac{1}{2 \pi} \int_{\Gamma} P_{t}(\xi)|f(\xi)-f(\tau)||d \xi|+|f(\tau)| .
$$

Therefore,

$$
|f(t)| \lesssim\left(|f(\tau)|+J_{\omega}\right)
$$

where

$$
J_{\omega}:=\frac{1}{2 \pi} \int_{\Gamma} P_{t}(\xi) \omega(f,|\xi-\tau|) d \tau .
$$


We proceed to estimating the latter integral. It is clear that

$$
J_{\omega} \lesssim \int_{\Gamma} \frac{\left(1-|t|^{2}\right) \omega(f,|\xi-\tau|)}{(1-|t|)^{2}+|\xi-\tau|^{2}}|d \xi| \lesssim \int_{0}^{\pi} \frac{\left(1-|t|^{2}\right) \omega(f, u)}{(1-|t|)^{2}+u^{2}} d u
$$
Then

We define $\omega(f, \delta)$ on $\mathbb{R}_{+}=[0,+\infty)$ as a function of modulus of continuity type (see [1], [4]).

$$
J_{\omega} \lesssim \int_{0}^{\frac{\pi}{1-|t|}} \frac{\omega(f, v(1-|t|))}{1+v^{2}} d v
$$

Representing this integral as the sum

$$
\int_{0}^{1} \frac{\omega(f, v(1-|t|))}{1+v^{2}} d v+\int_{1}^{\frac{\pi}{1-|t|}} \frac{\omega(f, v(1-|t|))}{1+v^{2}} d v
$$

and taking into consideration that $\frac{\omega(f, \delta)}{\delta}$ does not increase (see [1], [4]), we obtain

$$
J_{\omega} \lesssim \omega(f, 1-|t|) \int_{1}^{\frac{\pi}{1-|t|}} \frac{v}{1+v^{2}}
$$

Hence,

$$
J_{\omega} \lesssim \omega(f, 1-|t|) \ln \frac{1}{1-|t|}
$$

By (5), (6) we arrive at the statement of the lemma.

Lemma 3. (see [3]). Let $\lambda$ be a positive non-decreasing function on $(0,1)$ and

$$
\int_{0}^{1} \lambda(u) d u<+\infty
$$

Assume that $f$ is an analytic function in $D$ such that

$$
\sup _{z \in D}\left\{\frac{\left|f^{\prime}(z)\right|}{\lambda(|z|)}\right\}<+\infty
$$

Then the function $f$ belongs to the class $C_{A}$ and

$$
\omega(f, \delta) \lesssim \int_{1-\delta}^{1} \lambda(u) d u
$$

Lemma 4. Let $f \in C_{A}, f(z) \neq 0, z \in D,|f(z)| \leqslant 1, z \in D$. Then there exists a number $M>0$ possessing the following properties: for an arbitrary $0<a<1$, the function $f$ in $D$ can be represented as

$$
f(z)=\Phi_{a}(z) \Psi_{a}(z), z \in D
$$


where $\Psi_{a}$ is an analytic function in $D$ such that $\left|\Psi_{a}\right|$ is continuously extended to the entire closed circe $D \cup \Gamma$,

$$
\begin{aligned}
& a \leqslant\left|\Psi_{a}(t)\right| \leqslant 1 \quad \text { for each } t \in D, \\
& || \Psi_{a}\left(t^{\prime}\right)|-| \Psi_{a}\left(t^{\prime \prime}\right)|| \leqslant|| f\left(t^{\prime}\right)|-| f\left(t^{\prime \prime}\right)||, \quad t^{\prime}, t^{\prime \prime} \in \Gamma, \\
& \int_{\Gamma}|\ln | \Psi_{a}(t)|| d t \mid \leqslant M, \\
& \Phi_{a}(z)=\exp \left(-\int_{\Gamma} S_{z}(t) d \mu_{a}(t)\right), \quad z \in D,
\end{aligned}
$$

where $S_{z}(t)$ is the Schwarz kernel for the circle $D, \mu_{a}$ is a non-negative Borel measure $\Gamma$, whose total variation does not exceed $M$.

Proof. Let $H_{a}(t)=\max (a,|f(t)|), t \in \Gamma$. We have

$$
\begin{aligned}
& \Psi_{a}(z):=\exp \left(\frac{1}{2 \pi} \int_{\Gamma} S_{z}(t) \ln H_{a}(t)|d t|\right), \\
& \Phi_{a}(z)=f(z)\left(\Psi_{a}(z)\right)^{-1}:=\exp \left(-\int_{\Gamma} S_{z}(t) d \mu_{a}(t)\right), \quad z \in D,
\end{aligned}
$$

where

$$
\mu_{a}(E)=-\int_{E_{a}} \ln \left(\frac{|f(t)|}{a}\right)|d t|+\mu(E),
$$

$\mu$ is a non-negative measure defining the singular part of the function $f, E$ is an arbitrary Borel set in $\Gamma, E_{a}=\{\gamma \in \Gamma:|f(\gamma)| \leqslant a\}$. It is clear that $\left|\Psi_{a}\right|$ on $\Gamma$ coincides with $H_{a}$. Estimate (7) is obtained by the following inequality

$$
\begin{aligned}
\int_{\Gamma}|\ln | \Psi_{a}(\xi)|||d \xi| & =\int_{E(|f| \geqslant a)}|\ln | \Psi_{a}(\xi)|||d \xi|+\int_{E(|f|<a)}|\ln | \Psi_{a}(\xi)|||d \xi| \\
& =\int_{E(|f| \geqslant a)}|\ln | \Psi_{a}(\xi)|||d \xi|+\sigma\left(E_{a}\right) \ln \frac{1}{a},
\end{aligned}
$$

where $\sigma$ is the Lebesgue measure on $E_{a}$.

It remains to note that

$$
E_{a}=E(|f| \leqslant a)=E\left(\ln \frac{1}{|f|} \geqslant \ln \frac{1}{a}\right) ;
$$

re recall that $\max |f| \leqslant 1,0<a<1$.

The finiteness of the integral $\int_{\Gamma}|\ln | f(\xi)|||d \xi|$ implies that

$$
\sup _{A \geqslant 0} A \sigma(\gamma \in \Gamma:|\ln | f(\gamma)|| \geqslant A)<+\infty \text {. }
$$

This proves $(7)$.

Now we are going to estimate $\mu_{a}(E)$. In order to do it, we denote by $V_{a}(E)$, the first term in the right hand side in $(8)$ and we note that $\mu_{a}(\Gamma) \leqslant V_{a}(\Gamma)+\mu(\Gamma)$. This is why

$$
V_{a}(\Gamma) \leqslant \int_{\Gamma}|\ln | f|| d t+|\ln a| \sigma\left(\Gamma_{a}\right),
$$


where

$$
\Gamma_{a}=\{\gamma \in \Gamma:|\ln | f(\gamma)|| \geqslant|\ln a|\}
$$

Following the lines of the proof of inequality (7), we obtain the last statement of the lemma. The proof is complete.

Remark 3. Employing the Jensen inequality, it is easy to observe that if $\|f\|_{C_{A}}<1$, then

$$
\left|\mu_{a}(\Gamma)\right| \lesssim \ln \frac{1}{|f(0)|}, \quad \int_{\Gamma}|\ln | \Psi_{a}|||d t| \lesssim \ln \frac{1}{|f(0)|} .
$$

\section{ProOF OF THEOREMS}

Proof of Theorem 1. Without loss of generality we assume that $|f(t)| \leqslant 1, t \in \Gamma$. Moreover, for the sake of convenience we denote $\omega(\delta):=\omega(|f|, \delta), 0 \leqslant \delta \leqslant 2$, and at that,

$$
\left|f\left(t^{\prime}\right)\right|-\left|f\left(t^{\prime \prime}\right)\right| \leqslant \frac{1}{2} \omega\left(\left|t^{\prime}-t^{\prime \prime}\right|\right) \quad \text { for all } t^{\prime}, t^{\prime \prime} \in \Gamma .
$$

Employing Lemma 3, it is sufficient to establish the estimate

$$
\left|f^{\prime}(t)\right| \lesssim \frac{\omega(\sqrt{1-|t|})}{1-|t|}, \quad t \in D
$$

Let $t \in D$ be a fixed point in the circle $D$ and in Lemma 4 we choose $a=\omega(\sqrt{1-|t|})$.

We introduce the notations

$$
F_{t}(t)=\Psi_{\omega(\sqrt{1-|t|})}(t), \quad f_{t}(t)=\Phi_{\omega(\sqrt{1-|t|})}(t) .
$$

We observe that

$$
f^{\prime}(t)=f_{t}^{\prime}(t) F_{t}^{\prime}(t)+f_{t}^{\prime}(t) F_{t}(t)
$$

$1^{\circ}$. Estimate for $\left|f_{t}(t)\right|\left|F_{t}^{\prime}(t)\right|$. To estimate this product, let us first prove the inequality

$$
\left|F_{t}(t)\right| \lesssim\left|F_{t}(\tau)\right|, \quad t=|t| \tau, \quad \tau \in \Gamma .
$$

By Lemma 2,

$$
\left|F_{t}(t)\right| \lesssim\left(\left|F_{t}(\tau)\right|+\omega(1-|t|) \ln \frac{1}{1-|t|}\right)
$$

This is why to prove inequality (9), it is sufficient to establish the estimate

$$
\sup _{t \in D}\left\{\omega(1-|t|) \ln \frac{1}{1-|t|}\left|F_{t}^{-1}(\tau)\right|\right\}<+\infty .
$$

Suppose first that

$$
\max (|f(\tau)|, \omega(\sqrt{1-|t|}))=|f(\tau)|
$$

then by Lemma 4 we have

$$
\left|F_{t}(\tau)\right| \geqslant \omega(\sqrt{1-|t|})
$$

This is why, taking into consideration the estimate

$$
\omega(1-|t|) \ln \frac{1}{1-|t|} \lesssim \omega(\sqrt{1-|t|})
$$

we obtain

$$
\frac{\omega(1-|t|) \ln \frac{1}{1-|t|}}{\left|F_{t}(\tau)\right|} \lesssim \frac{\omega(\sqrt{1-|t|})}{\omega(\sqrt{1-|t|})} \lesssim 1 .
$$

Now we consider the case $|f(\tau)| \leqslant \omega(\sqrt{1-|t|})$. Applying Lemma 1 once again, we get desired estimate (9). 
We proceed to estimating the functions $\left|f_{t}(t)\right|,\left|F_{t}^{\prime}(t)\right|$. Let

$$
\Gamma_{1}=\left\{\gamma \in \Gamma: \omega(|\gamma-\tau|) \leqslant\left|F_{t}(\tau)\right|\right\}, \quad \Gamma_{2}=\Gamma \backslash \Gamma_{1} .
$$

Then we have

$$
\left|f_{t}(t)\right|\left|F_{t}^{\prime}(t)\right| \leqslant\left|F_{t}^{\prime}(t)\right|
$$

At that,

$$
\begin{aligned}
\left|F_{t}^{\prime}(t)\right| & =\left|F_{t}(t)\right|\left|\int_{\Gamma}\right| F_{t}(\gamma)\left|\frac{2 \gamma}{(\gamma-t)^{2}}\right| d \gamma|=| F_{t}(t)|| \int_{\Gamma} \frac{\left(\ln \left|F_{t}(\gamma)\right|-\ln \left|F_{t}(\tau)\right|\right)}{(\gamma-t)^{2}} \mid \\
& \left.\lesssim\left|F_{t}(\tau)\right||| \int_{\Gamma_{1}} \frac{\left(\ln \left|F_{t}(\gamma)\right|-\ln \left|F_{t}(\tau)\right|\right) \mid}{(\gamma-t)^{2}}|+| \int_{\Gamma_{2}} \frac{\left(\ln \left|F_{t}(\gamma)\right|-\ln \left|F_{t}(\tau)\right|\right)}{(\gamma-t)^{2}} \mid\right] \\
& \lesssim\left|F_{t}(\tau)\right| \int_{\Gamma_{1}} \frac{|\ln | F_{t}(\gamma)|-\ln | F_{t}(\tau)||}{|\gamma-t|^{2}}|d \gamma|+\left|F_{t}(\tau)\right| \int_{\Gamma_{2}} \frac{|\ln | F_{t}(\gamma)|||d \gamma|}{|\gamma-t|^{2}} \\
& +\left|F_{t}(\tau)\right||\ln | F_{t}(\tau)|| \int_{\Gamma_{2}} \frac{|d \gamma|}{|\gamma-t|^{2}} \stackrel{\text { def }}{=}\left[I_{1}+I_{2}+I_{3}\right] .
\end{aligned}
$$

Estimate for $I_{1}$. If $\gamma \in \Gamma_{1}$, by the mean theorem we have

$$
|\ln | F_{t}(\gamma)||-|\ln | F_{t}(\tau)|| \leqslant \frac{|| F_{t}(\gamma)|-| F_{t}(\tau)||}{\min _{\gamma \in \Gamma_{1}}\left(\left|F_{t}(\gamma)\right|,\left|F_{t}(\tau)\right|\right)} \leqslant \frac{|| f(\gamma)|-| f(\tau)||}{\min _{\gamma \in \Gamma_{1}}\left(\left|F_{t}(\gamma)\right|,\left|F_{t}(\tau)\right|\right)} .
$$

In view of the definition of $\Gamma_{1}$, we have

$$
\left|F_{t}(\gamma)\right| \geqslant|| F_{t}(\tau)|-| F_{t}(\tau)-F_{t}(\gamma)|| \geqslant\left|F_{t}(\tau)\right|-\frac{1}{2} \omega(|\gamma-\tau|) \geqslant \frac{1}{2}\left|F_{t}(\tau)\right| .
$$

Therefore,

$$
I_{1} \lesssim \frac{1}{1-|t|} \int_{\Gamma} \omega(|\gamma-\tau|) P_{t}(\gamma)|d \gamma|
$$

where $P_{t}(\gamma)$ is the Poisson kernel.

Employing Lemma 2, we obtain

$$
I_{1} \lesssim \frac{\omega(1-|t|)}{1-|t|} \ln \frac{1}{1-|t|}
$$

By Lemma 1, we finally get

$$
I_{1} \lesssim \frac{\omega(\sqrt{1-|t|)}}{1-|t|}, \quad t \in D
$$

Estimate for $I_{2}$. Let

$$
K_{t}(\gamma)=\frac{1}{(\gamma-t)^{2}}, \quad \gamma \in \Gamma, \quad t \in D
$$

Then we have

$$
I_{2} \leqslant\left|F_{t}(\tau)\right| \max _{\gamma \in \Gamma_{2}}\left|K_{t}(\gamma)\right| \int_{\Gamma}|\ln | f_{t}(\gamma)|||d \gamma| \lesssim\left|F_{t}(\tau)\right| \max _{t \in \Gamma_{2}}\left|K_{t}(\gamma)\right| .
$$

In the latter estimate we have employed Lemma 4.

Now, taking into consideration the definition of $\Gamma_{2}$, we obtain

$$
I_{2} \lesssim\left|F_{t}(\tau)\right| \max _{\gamma \in \Gamma_{2}} \frac{1}{\left(|\gamma-\tau|^{2}+(1-|t|)\right)^{2}} \lesssim\left|F_{t}(\tau)\right| \max \left\{\frac{1}{x^{2}}, x: \omega(x) \geqslant\left|F_{t}(\tau)\right|\right\} .
$$


Let $x^{*} \in(0,2]$ be such that

Then by the latter estimate we get

$$
\omega\left(x^{*}\right)=\left|F_{t}(\tau)\right|
$$

$$
I_{2} \lesssim \frac{\left|F_{t}(\tau)\right|}{\left(x^{*}\right)^{2}}=C_{f} \frac{\omega\left(x^{*}\right)}{\left(x^{*}\right)^{2}} .
$$

The inequality $\left|F_{t}(\tau)\right| \geqslant \omega(\sqrt{1-|t|})$ implies $\sqrt{1-|t|} \leqslant x^{*}$. This is why,

$$
\frac{\omega\left(x^{*}\right)}{\left(x^{*}\right)^{2}}=\frac{\omega\left(x^{*}\right)}{x^{*} \cdot x^{*}} \leqslant \frac{\omega(\sqrt{1-|t|})}{\sqrt{1-|t|}} \frac{1}{\sqrt{1-|t|}}=\frac{\omega(\sqrt{1-|t|})}{1-|t|},
$$

that is,

Estimate for $I_{3}$. We have

$$
I_{2} \lesssim \frac{\omega(\sqrt{1-|t|})}{(1-|t|)}
$$

$$
\begin{aligned}
I_{3} & =\left|F_{t}(\tau)\right||\ln | F_{t}(\tau)|| \int_{\Gamma_{2}} \frac{|d \gamma|}{|\gamma-t|^{2}} \lesssim\left|F_{t}(\tau)\right||\ln | F_{t}(\tau)|| \int_{\omega(u) \geqslant\left|F_{t}(\tau)\right|} \frac{d u}{u^{2}+(1-|t|)^{2}} \\
& =\left|F_{t}(\tau)\right||\ln | F_{t}(\tau)|| \lesssim \frac{\left|F_{t}(\tau)\right||\ln | F_{t}(\tau)||}{(1-|t|)}\left(\frac{\pi}{2}-\arg \cot \frac{x^{*}}{1-|t|}\right),
\end{aligned}
$$

where $x^{*}$ is introduced by identity (10).

Taking into consideration the elementary inequality

$$
0 \leqslant \frac{\pi}{2}-\arg \cot V \leqslant \frac{H}{1+V}, \quad V \in[0,+\infty),
$$

by estimate (11) we finally obtain

$$
I_{3} \lesssim\left|F_{t}(\tau)\right||\ln | F_{t}(\tau)|| \frac{H}{1-|t|+x^{*}} .
$$

Now, in view of the estimates

$$
\sup _{0 \leqslant u \leqslant 2} u|\ln u| \leqslant e, \quad x^{*} \geqslant \sqrt{1-|t|}
$$

by (11) we get

$$
I_{3} \lesssim \frac{1}{\sqrt{1-|t|}} \frac{\omega(\sqrt{1-|t|})}{\sqrt{1-|t|}}=C_{f} \frac{\omega(\sqrt{1-|t|})}{1-|t|} .
$$

In the latter inequality we employed the inequality $\frac{\omega(\delta)}{\delta} \geqslant \omega(1)$ as $0<\delta \leqslant 1$.

$2^{\circ}$. Estimate for $\left|F_{t}(\tau)\right|\left|f_{t}^{\prime}(t)\right|$ As above, we let

$$
K_{t}(\xi)=\frac{1}{(t-\xi)^{2}}, \quad \xi \in \Gamma, \quad t \in D
$$

Then

$$
f_{t}^{\prime}(t)=f_{t}(t) \int_{\Gamma} K_{t}(\xi) d \mu^{t}(\xi)
$$

where the measure $\mu^{t}$ is supported in the set

$$
E_{t}=\{\gamma \in \Gamma:|f(\gamma)| \leqslant \omega(\sqrt{1-|t|})\}
$$

at that, $\mu^{t}(\Gamma) \leqslant M$.

Let $\tau^{*} \in E_{t}$ be the point closest to the point $t$. Then by Lemma 2 we get

$$
\left|F_{t}(t)\right| \lesssim\left[\left|F_{t}(\tau)\right|+\omega(1-|t|) \ln \frac{1}{1-|t|}\right]
$$


Hence,

$$
\left|F_{t}(t)\right| \lesssim\left(\left|F_{t}(\tau)-F_{t}\left(\tau^{*}\right)\right|+\left|F_{t}\left(\tau^{*}\right)\right| \omega(1-|t|) \ln \frac{1}{1-|t|}\right) .
$$

Since $\tau^{*} \in E_{t}$, then $\left|F_{t}\left(\tau^{*}\right)\right| \leqslant \omega(\sqrt{1-|t|})$. Therefore, by estimate (12) we obtain

$$
\left|F_{t}(t)\right| \lesssim\left[\omega\left(\left|\tau-\tau^{*}\right|\right)+\omega(1-|t|) \ln \frac{1}{1-|t|}+\omega(\sqrt{1-|t|})\right] .
$$

By Lemma 1 we have

$$
\left|F_{t}(t)\right|\left|f_{t}^{\prime}(t)\right| \lesssim\left[\omega\left(\left|\tau-\tau^{*}\right|\right)+\omega(\sqrt{1-|t|})\right]\left|f_{t}(t)\right| \int_{\Gamma}\left|K_{t}(\xi)\right| d \mu^{t}(\xi),
$$

that is,

$$
\left|F_{t}(t)\right|\left|f_{t}^{\prime}(t)\right| \lesssim\left[\left|f_{t}^{\prime}(t)\right| \omega\left(\left|\tau-\tau^{*}\right|\right)\left|+f_{t}^{\prime}(t)\right| \omega(\sqrt{1-|t|})\right] .
$$

We proceed to estimating the expression in the brackets.

Let

$$
\begin{aligned}
& J_{1}=\left|f_{t}^{\prime}(t)\right| \omega\left(\left|\tau-\tau^{*}\right|\right)=\omega\left(\left|\tau-\tau^{*}\right|\right)\left|f_{t}(t)\right| \int_{\Gamma} \frac{d \mu^{t}(\xi)}{|\xi-t|^{2}}, \\
& J_{2}=\left|f_{t}^{\prime}(t)\right| \omega(\sqrt{1-|t|}) .
\end{aligned}
$$

We first estimate $J_{2}$.

We have

$$
\begin{aligned}
J_{2} & \lesssim \omega(\sqrt{1-|t|}) \int_{\Gamma} \frac{d \mu^{t}(\xi)}{|\xi-t|^{2}} \exp \left(-\frac{1-|t|^{2}}{|\xi-t|^{2}} d \mu^{t}(\xi)\right) \\
& \lesssim \frac{\omega(\sqrt{1-|t|})}{1-|t|} \sup _{u>0} e^{-u} u \lesssim \frac{\omega(\sqrt{1-|t|})}{1-|t|} .
\end{aligned}
$$

We proceed to estimating $J_{1}$. If $\omega\left(\left|\tau-\tau^{*}\right|\right) \lesssim \omega(\sqrt{1-|t|})$, then $J_{1}$ can be estimated exactly in the same way as $J_{2}$. This is why we assume that $\omega(\sqrt{1-|t|}) \leqslant \omega\left(\left|\tau-\tau^{*}\right|\right)$. In view of the monotonicity of the function $\omega$, the latter estimate implies $\sqrt{1-|t|}) \leqslant\left|\tau-\tau^{*}\right|$. Therefore, we obtain

$$
\begin{aligned}
J_{1} & \lesssim \omega\left(\left|\tau-\tau^{*}\right|\right)\left|f_{t}(t)\right| \frac{1}{\left|t-\tau^{*}\right|^{2}} \lesssim \frac{\omega\left(\left|\tau-\tau^{*}\right|\right)}{\left|t-\tau^{*}\right|} \frac{1}{\left|t-\tau^{*}\right|} \\
& \lesssim \frac{\omega\left(\left|\tau-\tau^{*}\right|\right)}{\left|\tau-\tau^{*}\right|} \frac{1}{\sqrt{1-|t|}} \lesssim \frac{\omega(\sqrt{1-|t|})}{(\sqrt{1-|t|})(\sqrt{1-|t|})}=\frac{\omega(\sqrt{1-|t|})}{1-|t|}, \quad t \in D .
\end{aligned}
$$

In the latter inequality we have employed the non-increasing of the function $\frac{\omega(\delta)}{\delta}$ on $(0,2)$.

Estimates (13), (14) imply the statement of the theorem.

Let us outline the proof of Theorem 2 .

Let $f$ satisfy the assumptions of Theorem 2. We consider the following cut-off function: $f(\lambda)=f(\lambda \xi), \lambda \in D, \xi \in S_{n}$, a point $\xi$ is fixed, see [11].

It is easy to see that $f_{\xi}(\lambda)$ satisfies the assumptions of Theorem 1. In view of Remark 2, we establish the estimate

$$
\left|f_{\xi}\left(\lambda_{1}\right)-f_{\xi}\left(\lambda_{2}\right)\right| \leqslant A \omega\left(\sqrt{\left|\lambda_{1}-\lambda_{2}\right|}\right), \quad \lambda_{1}, \lambda_{2} \in D,
$$

and $A$ is independent of $\xi \in S_{n}$. 
Employing the identity

$$
R(f)(z)=n \int_{S_{n}} d \sigma(\xi) \frac{1}{2 \pi} \int_{-\pi}^{\pi} \frac{\langle z, \xi\rangle e^{-i \theta} f\left(e^{i \theta} \xi\right) d \theta}{\left(1-\langle z, \xi\rangle e^{-i \theta}\right)^{n+1}}, \quad z \in B_{n},
$$

where $R$ is the radial derivative, see [11], we get the estimate

$$
|R(f)(z)| \lesssim \frac{\omega(\sqrt{1-\|z\|})}{1-\|z\|}, \quad z \in B_{n}
$$

Arguing as in the proof of Theorem 7.9 in [11], we arrive at the statement of Theorem 2.

\section{BIBLIOGRAPHY}

1. N.K. Bari, S.B. Stechkin. Best approximations and differential properties of two conjugate functions // Trudy Mosk. Matem. Obsch. 5, 482-522 (1956). (in Russian).

2. A.V. Vasin, S.V. Kislyakov, A.N. Medvedev. Local smoothness of an analytic function compared to the smoothness of its modulus // Alg. Anal. 25:3, 52-85 (2013). [St. Petersburg Math. J. 25:3, 397-420 (2013).]

3. Ya.L. Geronimus. On some properties of analytic functions continuous on a closed circle or circular sector // Matem. Sborn/ 38(80):3, 319-330 (1956). (in Russian).

4. N.P. Kornejchuk. Extremal problems of approximation theory. Nauka, Moscow (1976). (in Russian).

5. V.P. Havin, F.A. Shamoyan. Analytic functions with the boundary values having Lipschitz module // Zapis. Nauchn. Semin. LOMI. 19, 237-239 (1970). (in Russian).

6. V.P. Khavin. A genralization of the Privalov-Zygmund theorem on the modulus of continuity of the conjugate function // Izv. Akad. Nauk Arm. SSSR. Ser. Matem. 6, 252-258, 265-287 (1971).

7. F.A. Shamoyan. Some division problems in spaces of analytic functions. PhD thesis, Leningrad State Univ., Leningrad (1970). (in Russian).

8. N.A. Shirokov. Outer functions from the analytic O.V. Besov classes // Zapis. Nauch. Semin. POMI. 217, 172-217 (1994). [J. Math. Sci. 85:2, 1867-1897 (1997).]

9. N.A. Shirokov. Smoothness of a holomorphic function in a ball and smoothness of its modulus on the sphere // Zapis. Nauch. Semin. POMI. 447, 123-127 (2016). (in Russian).

10. N.A. Shirokov. Analytic functions smooth up to the boundary. Lect. Notes Math. 1312. Springer, Berlin (1988).

11. Kehe Zhu. Space of holomorphic functions in the unit ball. Graduate Texts Math. 226. Springer, Berlin (2005).

Faizo Agitovich Shamoyan,

Bryansk State University

named after Academician Ivan Georgiyevich Petrovsky

Bezhitsckaya str. 14,

241036, Bryansk, Russia

E-mail: shamoyanfa@yandex.ru 